\title{
Potential influencing factors of aortic diameter at specific segments in population with cardiovascular risk
}

\author{
Tingting Chen ${ }^{1}$, Xingan Yang ${ }^{4}$, Xiaoxin Fang ${ }^{5}$, Lijiang Tang ${ }^{1}$, Yang Zhang ${ }^{2,3}$, Yingzheng Weng ${ }^{1}$, \\ Hongliang Zhang ${ }^{2,3}$, Juntao $\mathrm{Wu}^{2,3}$, Ping Mao ${ }^{1}$, Baohui $\mathrm{Xu}^{6}$, Jianjun Jiang ${ }^{2,3}$ and Xiaofeng Chen ${ }^{2,3,7^{*}}$ (1)
}

\begin{abstract}
Background: Aortic diameter is a critical parameter for the diagnosis of aortic dilated diseases. Aortic dilation has some common risk factors with cardiovascular diseases. This study aimed to investigate potential influence of traditional cardiovascular risk factors and the measures of subclinical atherosclerosis on aortic diameter of specific segments among adults.

Methods: Four hundred and eight patients with cardiovascular risk factors were prospectively recruited in the observational study. Comprehensive transthoracic M-mode, 2-dimensional Doppler echocardiographic studies were performed using commercial and clinical diagnostic ultrasonography techniques. The aortic dimensions were assessed at different levels: (1) the annulus, (2) the mid-point of the sinuses of Valsalva, (3) the sinotubular junction, (4) the ascending aorta at the level of its largest diameter, (5) the transverse arch (including proximal arch, mid arch, distal arch), (6) the descending aorta posterior to the left atrium, and (7) the abdominal aorta just distal to the origin of the renal arteries. Multivariable linear regression analysis was used for evaluating aortic diameter-related risk factors, including common cardiovascular risk factors, co-morbidities, subclinical atherosclerosis, lipid profile, and hematological parameters.
\end{abstract}

Results: Significant univariate relations were found between aortic diameter of different levels and most traditional cardiovascular risk factors. Carotid intima-media thickness was significantly correlated with diameter of descending and abdominal aorta. Multivariate linear regression showed potential effects of age, sex, body surface area and some other cardiovascular risk factors on aortic diameter enlargement. Among them, high-density lipoprotein cholesterol had a significantly positive effect on the diameter of ascending and abdominal aorta. Diastolic blood pressure was observed for the positive associations with diameters of five thoracic aortic segments, while systolic blood pressure was only independently related to mid arch diameter.

Conclusion: Aortic segmental diameters were associated with diastolic blood pressure, high-density lipoprotein cholesterol, atherosclerosis diseases and other traditional cardiovascular risk factors, and some determinants still need to be clarified for a better understanding of aortic dilation diseases.

Keywords: Aortic diameter, Aorta dilation, Risk factors, Ultrasonography

*Correspondence: xiaof.chen@yahoo.com

${ }^{2}$ Department of Cardiology, Taizhou Hospital Affiliated to Wenzhou

Medical University, Linhai 317000, Zhejiang Province, China

Full list of author information is available at the end of the article

\section{Background}

Aorta dilation is a common problem in clinical practice, and the subsequent aortic aneurysm is a significant cause of adult death. The pathogenesis of aortic dilation 
is characterized by aortic wall inflammation, induction of smooth muscle cell apoptosis, extracellular matrix degradation, plaque formation, oxidative stress and vascular remodeling [1]. Potential biological mechanisms still need to be explored.

Aortic diameter is a critical parameter for the diagnosis of aortic aneurysms. The risk of rupture increases as the diameter of the aneurysm increases. Increased baseline diameter of infrarenal aorta is an independent risk factor for abdominal aortic aneurysm (AAA) in a populationbased follow-up study [2]. Increased aortic aneurysm diameter is associated with a significantly increased risk of future cardiovascular events and all-cause mortality [3], while previous follow-up research holds the same view for the whole range of diameter values [4]. Therefore, exploring the risk factors for aortic dilation has great significance in predicting, preventing and treating cardiovascular disease.

Currently, research on the epidemiology of aortic diameter is gradually emerging. Many studies pointed out that aortic diameter and cardiovascular diseases shared common influencing factors. Age, sex, race, body surface area (BSA), smoking status, alcohol consumption, and lipid profile, hypertension and diabetes mellitus (DM) are all known major traditional cardiovascular risk factors which have effects on aortic diameter [5-8]. Atherosclerosis is well-known as one of the main causes of aneurysm. AAA prevalence is significantly higher among patients with coronary artery disease (CAD) [9]. Limited research suggests that subclinical atherosclerosis and carotid atherosclerosis are positively related to aortic diameter growth $[10,11]$. However, there are inconsistent views among the current studies concerning the risk factors that affect the aorta diameter. Also, the segment-specific difference in the relationship between risk factors and aortic diameter has been indicated by previous research under different experimental conditions $[12,13]$. However, related research incorporated a small number of cardiovascular indicators and evaluated fewer aorta segments.

In the current study, in order to further explore the mechanism of the early stage of aortic dilation, we examined the association between nine segmental aortic diameters of non-aneurysmal aortas and cardiovascular risk factors and the measures of subclinical atherosclerosis.

\section{Methods}

\section{Study population}

A total of 520 consecutive hospitalized patients with cardiovascular risk factors were admitted from Taizhou Hospital affiliated to Wenzhou Medical University in China between December 2013 and December 2014 in the single-center observational study. Exclusion criteria were as follows: Marfan syndrome, aortic stenosis or aortic regurgitation more than mild in degree, active cancer, infective endocarditis, syphilitic aortitis, rheumatic heart disease, and valvular heart disease $(n=17)$. In addition, we excluded patients who couldn't clearly show the long axis of the aorta due to poor image quality $(\mathrm{n}=12)$ and patients with missing essential data $(n=83)$. Of the remaining, 408 patients participated in the final analysis. The study was in accordance with the Helsinki Declaration and approved by the Institutional Review Board. Informed consent was obtained from all participants.

\section{Baseline characteristic collection}

Information on the variables of interest like the patient's history of CAD, DM, hypertension, dyslipidemia, chronic obstructive pulmonary disease (COPD), cerebral infarction, medication history, and status of drinking and smoking could be collected after admission. Hypertension was defined as twice or more measured systolic blood pressure $(\mathrm{SBP}) \geq 140 \mathrm{mmHg}$ or diastolic blood pressure (DBP) $\geq 90 \mathrm{mmHg}$ measured at rest on the same day or current use of antihypertensive agents. DM was defined as currently using physician-prescribed hypoglycemic drugs or fasting serum glucose levels $>7 \mathrm{mmol} / \mathrm{L}$ and postprandial blood glucose $>11.1 \mathrm{mmol} / \mathrm{L}$. CAD referred to current or previous history of typical angina, acute coronary syndrome, or meeting the gold standard that detected coronary artery occlusion or stenosis by the coronary angiography technique. Hyperlipidemia was previously diagnosed with the plasma total cholesterol $>6.5 \mathrm{mmol} / \mathrm{L}$ or plasma triglyceride level $>1.7 \mathrm{mmol} / \mathrm{L}$ or current use of lipid-lowering therapy. Alcohol and smoking status were defined as YES or NO. Carotid intima-media thickness (CIMT), carotid atherosclerosis, carotid artery stenosis, and aortic sclerosis were detected by ultrasonography technique. SBP and DBP were measured on the seated subject's right arm in a calm state. The average of the second and third measurements was recorded. BSA was calculated according to the Stevenson formula: $\mathrm{BSA}\left(\mathrm{m}^{2}\right)=0.0061 \times$ height $(\mathrm{cm})+0.0128 \times$ weight $(\mathrm{kg})-0.1529$.

All patients received venous blood withdrawal after fasting overnight. Serum levels of total cholesterol (TC), high-density lipoprotein cholesterol (HDL-C), low-density lipoprotein (LDL-C), apolipoprotein A1, apolipoprotein $B$, lipoprotein (a), triglyceride, $C$-reactive protein (CRP), and hematological parameters including white blood cell (WBC) count, red blood cell (RBC) count, hemoglobin and platelet count, were detected in the clinic laboratory by commercially available standardized methods. 


\section{Evaluation of the aorta diameter by ultrasonography technique}

Comprehensive transthoracic M-mode, 2-dimensional Doppler echocardiographic studies were performed using a commercial and clinical diagnostic ultrasonography technique. Doppler echocardiographic examination was conducted on a GE Sonoline echocardiograph (GE, vivid E9, Boston, MA, USA) with a variable frequency probe from 2.5 to $5.5 \mathrm{MHz}$, and operated in strict accordance with the guidelines of the echocardiograph. The aortic dimensions were assessed at different levels: (1) the annulus, (2) the mid-point of the sinuses of Valsalva, (3) the sinotubular junction, (4) the ascending aorta at the level of its largest diameter, (5) the transverse arch including proximal arch, mid arch and distal arch, (6) the descending aorta posterior to the left atrium, and (7) the abdominal aorta just distal to the origin of the renal arteries. The aortic diameter was measured using the inner-inner line method, and the largest diameter was reported. The aortic root and the ascending aorta were taken on the parasternal long-axis view. The aortic arch measurement was taken on the superior sternal fossa long-axis view. The descending aorta was measured along the short-axis of the left ventricle next to the sternum. The abdominal aorta was measured along the long axis under the xiphoid process [14]. Except for the measurement of the aortic annulus in the mid-systole, the rest of the aortic segments were measured in the end-diastole, making sure that the measurement line was perpendicular to the long axis of the aorta. Measurements were averaged from 3 to 5 beats. The measurement was completed by two professional technicians with more than five years of work experience and the measurement data was completed by one of the technicians.

\section{Statistical analyses}

Continuous variables that conformed to normal distribution were expressed as mean values, and its standard deviation, and that non-normally distributed the median and its range represented variables. Categorical variables were expressed in percentiles. Differences between the two groups were assessed using independent samples T-test or Mann-Whitney U-test for continuous variables according to whether the data obeys normality distribution. The chi-square test was for categorical variables. Baseline variables (including sex, age, BSA, smoking, drinking, SBP, DBP, hypertension, DM, CAD, hyperlipidaemia, triglyceride, HDL-C, LP (a), statin agent, hypoglycemic agent and anti-hypertension agent) that were considered clinically relevant or showed a univariate relationship with the aortic diameter with $P<0.1$ were entered into multivariate linear regression analysis.
Variables for inclusion were carefully chosen, given the number of events available, to ensure the parsimony of the final models. An unstandardized regression coefficient was used to represent an increase or decrease in the outcome as the variables change. Variance inflation factor (VIF) was applied to evaluate the collinearity in the multiple regression model. Variables with a $\mathrm{VIF} \geq 3$, the index for the presence of collinearity, have been excluded from our analysis, including TC, LDL-C, apolipoprotein A1 and apolipoprotein B. Values of $P<0.05$ were considered statistically significant. Data analysis and processing were performed by SPSS (version 20.0) package.

\section{Results}

\section{Baseline characteristics}

Consecutive 408 patients were analyzed in the study. The average age of participants was median 66 years (range from 40 to 92 years old), and 36\% (147) were female. The data (Table 1) showed no difference in age, DBP, serum levels of lipoprotein(a) and CRP, WBC count, use of antiplatelet, statins and antihypertensive agents, as well as the percentage of DM, hyperlipidaemia, carotid atherosclerosis, carotid artery stenosis and aortic sclerosis, between male and female groups. Meanwhile, SBP, serum lipid profile levels including TC, triglyceride, HDL-C, LDL-C and platelet count in females, were significantly higher than those in males. On the contrary, BSA, RBC count, hemoglobin and CIMT, alcohol and smoking status, use of hypoglycemic agents, as well as the presence of CAD, hypertension, cerebral infarction and COPD in males were greater than those in females. The aortic diameter of each segment was reported in Table 2. Almost all aortic segment diameters are significantly larger in the male group than in the female group, except for ascending aorta $(P=0.078)$, as illustrated in Fig. 1.

\section{Aortic diameter and cardiovascular risk factors}

As displayed in Tables 3, 4 and 5, univariate analysis (Table 3) suggested that the diameters of the annulus, sinuses of Valsalva and sinotubular junction were positively correlated with sex, BSA, smoking, drinking, DBP, $\mathrm{RBC}$ count and hemoglobin. Among them only annulus diameter was negatively correlated with age and DM. As for aortic arch segments (Table 4), the diameter of all three segmental arches were positively correlated with sex, BSA, smoking, DBP, RBC count and hemoglobin, and were negatively correlated hypoglycemic agents use. Unlike the adjacent segments, ascending aorta diameter was positively correlated with age, BSA, SBP, DBP, hypertension, hemoglobin and antihypertension agents use, and negatively correlated with statin agents use. The diameter of descending aorta and abdominal aorta had some common influencing factors 
Table 1 Characteristic of participants

\begin{tabular}{|c|c|c|c|c|}
\hline Variables & $\begin{array}{l}\text { All } \\
\text { Mean } \pm \text { SD or median or } \\
\mathbf{n}(\%)\end{array}$ & $\begin{array}{l}\text { Male } \\
\text { Mean } \pm \text { SD or median or } \\
\mathbf{n}(\%)\end{array}$ & $\begin{array}{l}\text { Female } \\
\text { Mean } \pm \text { SD or median or } \\
\mathbf{n}(\%)\end{array}$ & $P$ value \\
\hline Age, year & $66(40-92)$ & $66(40-91)$ & $68(41-92)$ & 0.173 \\
\hline BSA & $1.65(1.32-2.14)$ & $1.70(1.35-2.14)$ & $1.52(1.32-1.96)$ & 0.000 \\
\hline Smoking, n (\%) & $161(39.5 \%)$ & $157(60.2 \%)$ & $4(2.7 \%)$ & 0.000 \\
\hline Alcohol, n (\%) & $86(21.1 \%)$ & $85(32.6 \%)$ & $1(0.7 \%)$ & 0.000 \\
\hline $\mathrm{SBP}, \mathrm{mmHg}$ & $149.3 \pm 24.6$ & $146.2 \pm 24.2$ & $154.9 \pm 24.5$ & 0.001 \\
\hline $\mathrm{DBP}, \mathrm{mmHg}$ & $83.3 \pm 13.7$ & $82.9 \pm 14.0$ & $83.8 \pm 13.2$ & 0.533 \\
\hline CAD, n (\%) & $175(42.9 \%)$ & $132(50.6 \%)$ & $43(29.3 \%)$ & 0.000 \\
\hline Hypertension, n (\%) & $268(65.7 \%)$ & $160(61.3 \%)$ & $108(73.5 \%)$ & 0.013 \\
\hline $\mathrm{DM}, \mathrm{n}(\%)$ & $97(23.8 \%)$ & $55(21.1 \%)$ & $42(28.6 \%)$ & 0.088 \\
\hline Hyperlipidaemia, n (\%) & $24(5.9 \%)$ & $16(6.1 \%)$ & $8(5.4 \%)$ & 0.777 \\
\hline Cerebral infarction, n (\%) & $183(44.9 \%)$ & $107(41 \%)$ & $76(51.7 \%)$ & 0.037 \\
\hline COPD, n (\%) & $15(3.7 \%)$ & $14(5.4 \%)$ & $1(0.7 \%)$ & 0.016 \\
\hline Carotid atherosclerosis, n (\%) & $251(61.5 \%)$ & $164(62.8 \%)$ & $87(59.2 \%)$ & 0.467 \\
\hline Carotid artery stenosis, n (\%) & $50(12.3 \%)$ & $35(13.4 \%)$ & $15(10.2 \%)$ & 0.344 \\
\hline Aortic sclerosis, n (\%) & $146(35.8 \%)$ & $101(38.7 \%)$ & $45(30.6 \%)$ & 0.102 \\
\hline CIMT, mm & $1.50(0.5-6.7)$ & $1.70(0.6-6.7)$ & $1.30(0.5-5.0)$ & 0.000 \\
\hline Triglyceride, mmol/L & $1.47(0.35-8.87)$ & $1.35(0.43-8.87)$ & $1.6(0.35-8.51)$ & 0.010 \\
\hline $\mathrm{TC}, \mathrm{mmol} / \mathrm{L}$ & $4.40(0.52-8.58)$ & $4.25(0.52-7.95)$ & $4.62(2.34-8.58)$ & 0.000 \\
\hline $\mathrm{HDL}-\mathrm{C}, \mathrm{mmol} / \mathrm{L}$ & $1.19(0.54-2.40)$ & $1.15(0.64-2.32)$ & $1.26(0.54-2.40)$ & 0.000 \\
\hline $\mathrm{LDL}-\mathrm{C}, \mathrm{mmol} / \mathrm{L}$ & $2.49(0.87-6.47)$ & $2.41(0.87-5.81)$ & $2.66(1.01-6.47)$ & 0.003 \\
\hline Lipoprotein (a), mg/L & $194(15-1398)$ & $186(24-1214)$ & $213(15-1398)$ & 0.889 \\
\hline $\mathrm{CRP}, \mathrm{mg} / \mathrm{L}$ & $4.1(1-189)$ & $4.3(1-164)$ & $3.4(1-189)$ & 0.254 \\
\hline WBC count & $6.6(3-19.2)$ & $6.63(3-19.2)$ & $6.4(3.6-17.3)$ & 0.174 \\
\hline Platelet count & $207(68-576)$ & $193(68-424)$ & $234(81-576)$ & 0.000 \\
\hline RBC & $4.4(2.1-15.9)$ & $4.5(2.1-15.9)$ & $4.2(3.07-5.65)$ & 0.000 \\
\hline Hemoglobin, g/L & $132(66-188)$ & $138(66-188)$ & $126(85-152)$ & 0.000 \\
\hline Statin agent, n (\%) & $103(25.2 \%)$ & $67(25.7 \%)$ & $36(24.5 \%)$ & 0.792 \\
\hline Anti-platelets agent, n (\%) & $222(54.4 \%)$ & $150(57.5 \%)$ & $72(49 \%)$ & 0.099 \\
\hline Anti-hypertension agent, n (\%) & $194(47.5 \%)$ & $118(45.2 \%)$ & $76(51.7 \%)$ & 0.208 \\
\hline Hypoglycemic agent, n (\%) & $56(13.7 \%)$ & $29(11.1 \%)$ & $27(18.4 \%)$ & 0.041 \\
\hline
\end{tabular}

Statistical differences: $P<0.05$

$B S A$ body surface area, $S B P$ systolic blood pressure, $D B P$ diastolic blood pressure, $C A D$ coronary artery disease, $D M$ diabetes mellitus, $C O P D$ chronic obstructive pulmonary disease, CIMT carotid intima-media thickness, $T C$ total cholesterol, $H D L$ - $C$ high-density lipoprotein cholesterol, $L D L-C$ low-density lipoprotein cholesterol, CRP C-reaction protein, WBC white blood cell

Table 2 Aortic diameter of each segment

\begin{tabular}{|c|c|c|c|c|}
\hline Aortic diameter & $\begin{array}{l}\text { All } \\
\text { Mean } \pm \text { SD or median or } n(\%)\end{array}$ & $\begin{array}{l}\text { Male } \\
\text { Mean } \pm \text { SD or median or } n(\%)\end{array}$ & $\begin{array}{l}\text { Female } \\
\text { Mean } \pm \text { SD or median or } n(\%)\end{array}$ & $P$ value \\
\hline Anulus & $19.3(15.6-24.7)$ & $19.9(16.2-24.7)$ & $18.4(15.6-22.4)$ & 0.000 \\
\hline Sinuses of Valsalva & $32.1(24.7-42.0)$ & $33.4(25.8-42.0)$ & $30.1(24.7-38.6)$ & 0.000 \\
\hline Sinotubular junction & $25.1(19.0-34.3)$ & $25.8(19-34.3)$ & $23.9(19.3-29.6)$ & 0.000 \\
\hline Proximal arch & $28.7 \pm 2.4$ & $29.0 \pm 2.41$ & $28.1 \pm 2.3$ & 0.000 \\
\hline Mid arch & $23.4 \pm 2.3$ & $23.8 \pm 2.3$ & $22.7 \pm 2.1$ & 0.000 \\
\hline Distal arch & $20.1 \pm 1.9$ & $20.5 \pm 1.8$ & $19.6 \pm 1.8$ & 0.000 \\
\hline Ascending aorta & $32.0(24.8-42.3)$ & $32.2(25.1-42.3)$ & $31.8(24.8-42.1)$ & 0.078 \\
\hline Descending aorta & $19.5 \pm 2.1$ & $19.9 \pm 2.1$ & $18.7 \pm 1.9$ & 0.000 \\
\hline Abdominal aorta & $15.2 \pm 1.7$ & $15.6 \pm 1.7$ & $14.5 \pm 1.4$ & 0.000 \\
\hline
\end{tabular}

Statistical differences: $p<0.05$ 

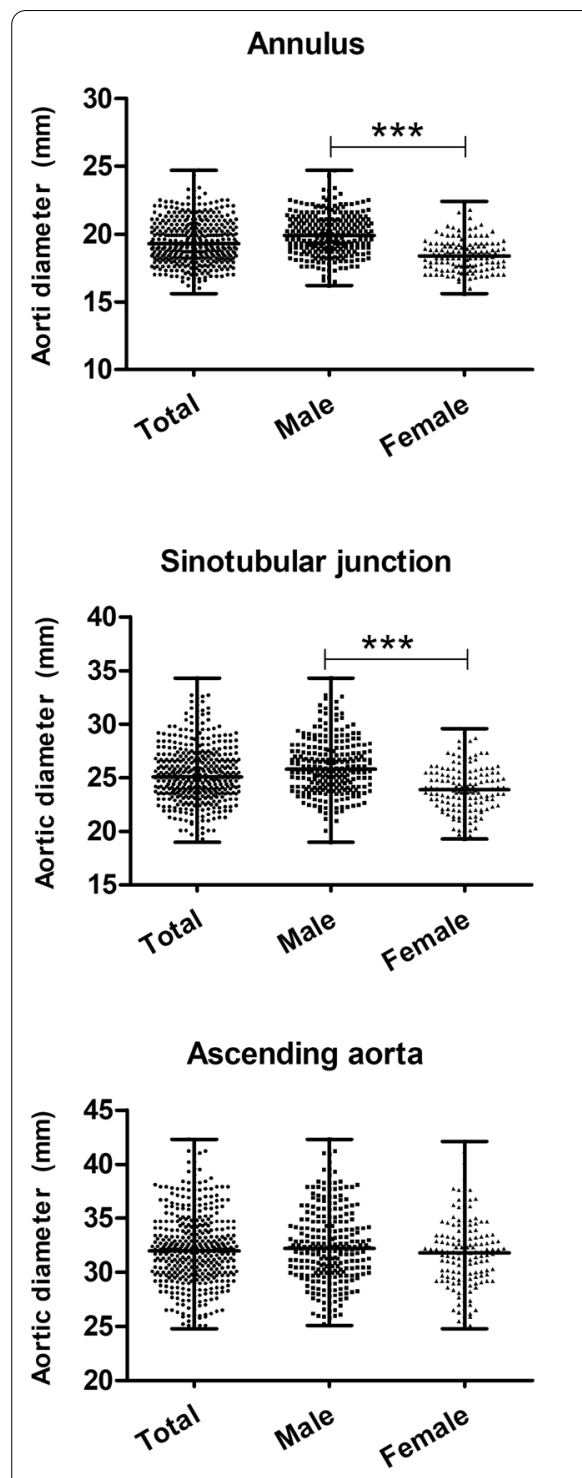

Fig. 1 Sex difference in each level of aortic dimension
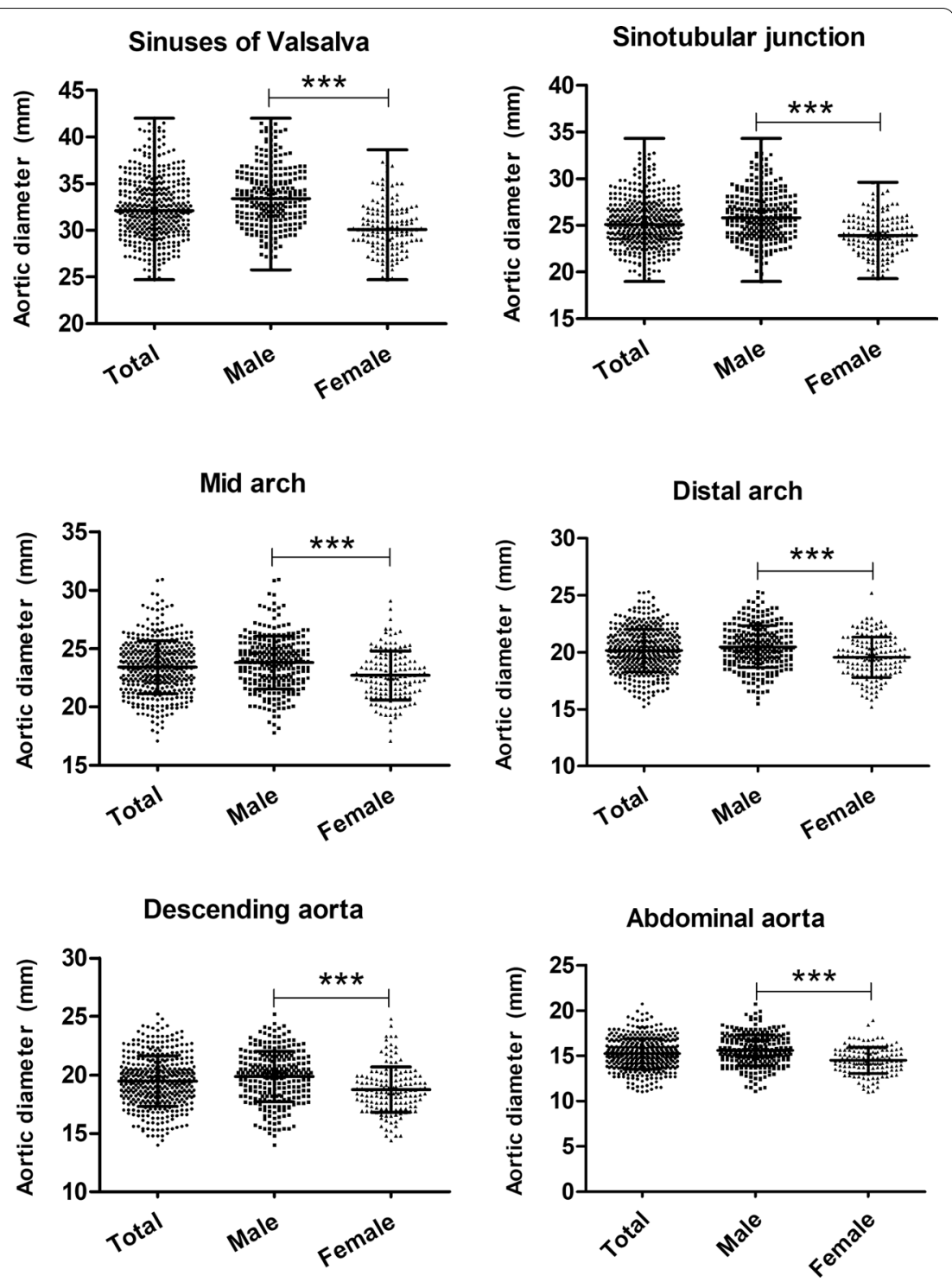

(Table 5). Positively related factors included sex, BSA, smoking, drinking, CIMT and hemoglobin. Aortic diameters of all nine segments were significantly negatively related to the use of hypoglycemic agent.

Inconsistent with the unadjusted regression, as showed in Tables 6, 7 and 8, multivariable-adjusted association revealed significant increases in diameters with age $(P<0.05)$ in segments of mid arch $(\beta=0.026, P=0.024)$, distal arch $(\beta=0.021, \quad P=0.030)$, ascending aorta $(\beta=0.066, P<0.001)$ and descending aorta $(\beta=0.039$, $P<0.001)$. The diameter of annulus $(\beta=0.891, P<0.001)$, sinuses of Valsalva $(\beta=2.128, P<0.001)$, sinotubular junction $(\beta=1.320, P=0.001)$, mid arch $(\beta=0.810$, $P=0.016)$, distal arch $(\beta=0.734, \quad P=0.007)$ and abdominal aorta $(\beta=0.845, P=0.001)$ were significantly increased in males. BSA was positively associated with the diameter of the annulus $(\beta=1.673, P=0.008)$, sinuses of Valsalva $(\beta=3.793, P=0.005)$, sinotubular junction $(\beta=2.253, P=0.041)$, ascending aorta $(\beta=3.429$, $P=0.018)$, descending aorta $(\beta=3.253, \quad P<0.001)$. Five of nine segments including sinotubular junction $(\beta=0.025, P=0.039)$, mid arch $(\beta=0.046, P<0.001)$, distal arch $(\beta=0.038, P<0.001)$, ascending aorta $(\beta=0.064$, $P<0.001)$ and descending aorta $(\beta=0.032, P=0.001)$ were observed for the positive associations of DBP with aortic diameters. SBP was only observed for the inverse association with the mid arch diameter $(\beta=-0.019$, $P=0.002)$. Differently, hypertension was observed for its 
Table 3 Correlation between the aortic diameters of annulus, sinuses of Valsalva, sinotubular junction and risk factors by univariate regression analysis

\begin{tabular}{|c|c|c|c|c|c|c|}
\hline \multirow[t]{2}{*}{ Variables } & \multicolumn{2}{|l|}{ Annulus } & \multicolumn{2}{|c|}{ Sinuses of Valsalva } & \multicolumn{2}{|c|}{ Sinotubular junction } \\
\hline & Coefficient $\rho$ & $P$ values & Coefficient $\rho$ & $P$ values & Coefficient $\rho$ & $P$ values \\
\hline Sex & 1.487 & 0.000 & 3.257 & 0.000 & 2.054 & 0.000 \\
\hline Age, year & -0.031 & 0.000 & -0.002 & 0.900 & -0.024 & 0.050 \\
\hline BSA & 4.340 & 0.000 & 8.596 & 0.000 & 5.707 & 0.000 \\
\hline Smoking & 1.115 & 0.000 & 2.033 & 0.000 & 1.279 & 0.000 \\
\hline Drinking & 0.601 & 0.002 & 1.549 & 0.000 & 0.754 & 0.019 \\
\hline SBP & -0.001 & 0.841 & 0.014 & 0.038 & 0.005 & 0.361 \\
\hline DBP & 0.011 & 0.049 & 0.032 & 0.009 & 0.027 & 0.004 \\
\hline Hypertension & -0.254 & 0.124 & 0.087 & 0.808 & 0.075 & 0.788 \\
\hline $\mathrm{DM}$ & -0.471 & 0.010 & -0.740 & 0.063 & -0.364 & 0.248 \\
\hline$C A D$ & 0.146 & 0.357 & -0.067 & 0.833 & -0.407 & 0.189 \\
\hline Hyperlipidaemia & -0.168 & 0.614 & -0.152 & 0.833 & -0.370 & 0.510 \\
\hline Triglyceride, $\mathrm{mmol} / \mathrm{L}$ & 0.032 & 0.607 & -0.069 & 0.610 & 0.086 & 0.410 \\
\hline $\mathrm{TC}, \mathrm{mmol} / \mathrm{L}$ & 0.020 & 0.780 & -0.171 & 0.259 & -0.205 & 0.081 \\
\hline $\mathrm{HDL}-\mathrm{C}, \mathrm{mmol} / \mathrm{L}$ & -0.146 & 0.632 & -0.222 & 0.736 & 0.109 & 0.832 \\
\hline $\mathrm{LDL}-\mathrm{C}, \mathrm{mmol} / \mathrm{L}$ & 0.001 & 0.987 & -0.165 & 0.399 & -0.230 & 0.131 \\
\hline Lipoprotein (a) & -0.001 & 0.084 & -0.001 & 0.324 & -0.001 & 0.269 \\
\hline COPD & - & - & 1.555 & 0.084 & - & - \\
\hline Arteriosclerosis & -0.274 & 0.094 & - & - & - & - \\
\hline $\mathrm{RBC}$ & 0.319 & 0.002 & 0.461 & 0.036 & 0.340 & 0.047 \\
\hline Hemoglobin & 0.030 & 0.000 & 0.065 & 0.000 & 0.043 & 0.000 \\
\hline Statin agent & -0.141 & 0.435 & -0.100 & 0.797 & -0.388 & 0.201 \\
\hline Hypoglycemic agent & -0.784 & 0.001 & -1.551 & 0.002 & -0.915 & 0.017 \\
\hline Anti-hypertension agent & -0.159 & 0.311 & 0.294 & 0.387 & 0.243 & 0.357 \\
\hline
\end{tabular}

Statistical differences: $P<0.05$; only baseline variables and other interested variables that showed a univariate relationship with aortic diameter with $P<0.1$ were displayed in the table

$B S A$ body surface area, $S B P$ systolic blood pressure, $D B P$ diastolic blood pressure, $C A D$ coronary artery disease, $D M$ diabetes mellitus, $C O P D$ chronic obstructive pulmonary disease, $T C$ total cholesterol, $H D L-C$ high-density lipoprotein cholesterol, $L D L-C$ low-density lipoprotein cholesterol, $R B C$ red blood cell

- Not applicable

inverse association with the diameter of proximal arch $(\beta=0.803, P=0.023)$, mid arch $(\beta=0.781, P=0.016)$ and descending aorta $(\beta=0.661, P=0.030)$. CAD had a significant reverse relationship with the diameter of transverse arch $(\beta=-1.064, P<0.001 ; \beta=-1.068, P<0.001$; $\beta=-0.478, P=0.017)$ and abdominal aorta $(\beta=-0.489$, $P=0.009$ ). Lipid profile had no relation to any aortic segments in unadjusted regression, while HDL-C turned to have a significantly positive effect on the diameter of ascending $(\beta=1.348, P=0.042)$ and abdominal aorta $(\beta=0.947, P=0.004)$. The use of hypoglycemic drugs was independently negatively associated with the diameter of transverse arch $(\beta=-1.105, P=0.023$; $\beta=-0.954, P=0.032 ; \beta=-0.919, P=0.011)$, descending aorta $(\beta=-0.923, P=0.026)$ and abdominal aorta $(\beta=-0.666, P=0.047)$.

\section{Ascending aorta diameter with no dilation and cardiovascular risk factors}

Ascending aortic dilation was considered in 38 patients with diameter greater than $37 \mathrm{~mm}$. We conducted an additional multivariate linear regression excluding the participants with ascending aortic dilation, and found that age $(\beta=0.055, P<0.001)$, BSA $(\beta=2.746, P=0.030)$, and diastolic blood pressure $(\beta=0.034, P=0.016)$ were independent positively associated with ascending aortic diameter with no dilation (Table 9).

\section{Discussion}

The current research indicated that some cardiovascular risk factors such as HDL-C, SBP, DBP. BSA may have an effect on increasing the diameter of the aorta in a specific segment. Also, to explore the risk factors of ascending aorta expansion, we found that age, BSA and DBP were 
Table 4 Correlation between the aortic diameters of aortic arch and risk factors by univariate regression analysis

\begin{tabular}{|c|c|c|c|c|c|c|}
\hline \multirow[t]{2}{*}{ Variables } & \multicolumn{2}{|l|}{ Proximal arch } & \multicolumn{2}{|l|}{ Mid arch } & \multicolumn{2}{|l|}{ Distal arch } \\
\hline & Coefficient $\rho$ & $P$ values & Coefficient $\rho$ & $P$ values & Coefficient $\rho$ & $P$ values \\
\hline Sex & 0.940 & 0.000 & 1.092 & 0.000 & 0.927 & 0.000 \\
\hline Age, year & 0.006 & 0.592 & 0.004 & 0.718 & 0.012 & 0.152 \\
\hline BSA & 2.816 & 0.000 & 2.796 & 0.000 & 1.860 & 0.002 \\
\hline Smoking & 0.800 & 0.001 & 0.631 & 0.006 & 0.457 & 0.015 \\
\hline Alcohol & 0.714 & 0.015 & 0.359 & 0.191 & 0.520 & 0.021 \\
\hline SBP & 0.013 & 0.007 & 0.007 & 0.118 & 0.010 & 0.010 \\
\hline DBP & 0.030 & 0.000 & 0.037 & 0.000 & 0.033 & 0.000 \\
\hline Hypertension & 0.771 & 0.002 & 0.605 & 0.010 & 0.373 & 0.055 \\
\hline DM & -0.391 & 0.165 & -0.571 & 0.029 & -0.845 & 0.000 \\
\hline$C A D$ & -0.750 & 0.002 & -0.701 & 0.002 & -0.357 & 0.056 \\
\hline Hyperlipidaemia & 0.591 & 0.247 & -0.057 & 0.905 & 0.134 & 0.733 \\
\hline Triglyceride & -0.041 & 0.662 & -0.008 & 0.928 & -0.117 & 0.109 \\
\hline $\mathrm{TC}, \mathrm{mmol} / \mathrm{L}$ & -0.028 & 0.792 & -0.134 & 0.179 & -0.042 & 0.615 \\
\hline $\mathrm{HDL}-\mathrm{C}, \mathrm{mmol} / \mathrm{L}$ & -0.271 & 0.560 & 0.115 & 0.790 & 0.268 & 0.455 \\
\hline $\mathrm{LDL}-\mathrm{C}, \mathrm{mmol} / \mathrm{L}$ & 0.045 & 0.747 & -0.232 & 0.071 & -0.030 & 0.779 \\
\hline Lipoprotein (a) & 0.000 & 0.445 & -0.001 & 0.161 & 0.000 & 0.692 \\
\hline Cerebral infarction & - & - & 0.405 & 0.072 & - & - \\
\hline Carotid atherosclerosis & - & - & - & - & 0.339 & 0.075 \\
\hline $\mathrm{RBC}$ & 0.421 & 0.007 & 0.407 & 0.005 & 0.288 & 0.016 \\
\hline Hemoglobin & 0.029 & 0.000 & 0.027 & 0.000 & 0.017 & 0.004 \\
\hline Statin agent & -0.502 & 0.069 & -0.392 & 0.128 & -0.056 & 0.793 \\
\hline Hypoglycemic agent & -1.044 & 0.003 & -1.123 & 0.001 & -1.391 & 0.000 \\
\hline Anti-hypertension agent & 0.576 & 0.016 & 0.447 & 0.046 & 0.328 & 0.076 \\
\hline
\end{tabular}

Statistical differences: $P<0.05$; only baseline variables and other interested variables that showed a univariate relationship with aortic diameter with $P<0.1$ were displayed in the table

$B S A$ body surface area, $S B P$ systolic blood pressure, $D B P$ diastolic blood pressure, $C A D$ coronary artery disease, $D M$ diabetes mellitus, $C O P D$ chronic obstructive pulmonary disease, CIMT carotid intima-media thickness, TC Total cholesterol, HDL-C high-density lipoprotein cholesterol, $L D L-C$ low-density lipoprotein cholesterol, $R B C$ red blood cell

- Not applicable

independent positively influencing factors of ascending aortic diameter with no dilation.

Aortic diameter is an important indicator of aortic dilation disease. According the studies focused on different aortic segments, there is an apparent overlap between the influencing factors of the aortic diameter and cardiovascular risk factors $[12,13,15]$, which can also be reflected in our current research.

In agreement with the previous research $[12,16]$, the present study shows a link between aortic diameter enlargement and aging, sex and BSA in some aortic segments. The aorta size increases throughout life, accompanied by loss of compliance and increased wall stiffness, leading to arterial dilation. A population-based study of Germany, Hatemi et al. [12] found that the diameter of each segment of the thoracic aorta and abdominal aorta expands with age, but we failed to show a statistical relationship in the abdominal aorta. Sex difference in aortic diameter and the risk of aortic diseases is a well-known fact [17]. The current results suggested that the diameters of almost all aortic segments were higher in males except for ascending aorta $(P=0.078)$. However, our multivariable model did not find an independent association between sex and the diameter of ascending aorta, descending aorta and proximal arch. At the same time, the confirmatory association of aortic diameter with BSA was only reflected in thoracic aorta segments in current results, supported by prior studies[16, 17].

Previous studies reported contradictory results about the role of high blood pressure as a major risk factor for aortic dilation. In a general population [12], DBP was positively and SBP was reversely associated with thoracic and abdominal aortic diameters. Glauser et al. [10] and Vasan et al. [18] both reported a completely consistent view in aortic root and abdominal aorta. However, in a cardiovascular risk screening program conducted in Paris [19], ascending aortic diameter was positively associated with SBP and DBP after excluding participants 
Table 5 Correlation between the aortic diameters of ascending aorta, descending aorta, abdominal aorta and risk factors by univariate regression analysis

\begin{tabular}{|c|c|c|c|c|c|c|}
\hline \multirow[t]{2}{*}{ Variables } & \multicolumn{2}{|c|}{ Ascending aorta } & \multicolumn{2}{|c|}{ Descending aorta } & \multicolumn{2}{|c|}{ Abdominal aorta } \\
\hline & Coefficient $\rho$ & $P$ values & Coefficient $\rho$ & $P$ values & Coefficient $\rho$ & $P$ values \\
\hline Sex & 0.664 & 0.055 & 1.135 & 0.000 & 1.100 & 0.000 \\
\hline Age, year & 0.043 & 0.005 & 0.019 & 0.056 & $-6.926 E-005$ & 0.993 \\
\hline BSA & 2.828 & 0.010 & 4.044 & 0.000 & 2.454 & 0.000 \\
\hline Smoking & 0.230 & 0.499 & 0.755 & 0.000 & 0.675 & 0.000 \\
\hline Alcohol & 0.393 & 0.335 & 0.607 & 0.019 & 0.727 & 0.000 \\
\hline SBP & 0.022 & 0.001 & 0.009 & 0.045 & 0.003 & 0.383 \\
\hline DBP & 0.051 & 0.000 & 0.025 & 0.001 & 0.012 & 0.054 \\
\hline Hypertension & 1.204 & 0.001 & 0.631 & 0.005 & 0.271 & 0.126 \\
\hline DM & -0.072 & 0.853 & -0.334 & 0.180 & -0.262 & 0.184 \\
\hline CAD & -0.206 & 0.539 & -0.102 & 0.633 & -0.212 & 0.212 \\
\hline Hyperlipidaemia & 0.561 & 0.427 & -0.128 & 0.777 & 0.241 & 0.501 \\
\hline Triglyceride, $\mathrm{mmol} / \mathrm{L}$ & -0.156 & 0.237 & -0.010 & 0.777 & -0.035 & 0.600 \\
\hline $\mathrm{TC}, \mathrm{mmol} / \mathrm{L}$ & -0.024 & 0.872 & -0.051 & 0.593 & -0.051 & 0.500 \\
\hline $\mathrm{HDL}-\mathrm{C}, \mathrm{mmol} / \mathrm{L}$ & 1.090 & 0.090 & -0.484 & 0.239 & 0.516 & 0.113 \\
\hline $\mathrm{LDL}-\mathrm{C}, \mathrm{mmol} / \mathrm{L}$ & 0.042 & 0.828 & -0.017 & 0.892 & -0.052 & 0.591 \\
\hline Lipoprotein (a) & 0.000 & 0.547 & -0.001 & 0.249 & 0.000 & 0.371 \\
\hline Carotid atherosclerosis & - & - & - & - & 0.311 & 0.072 \\
\hline CIMT & - & - & 0.252 & 0.018 & 0.190 & 0.024 \\
\hline CRP & - & - & -0.010 & 0.015 & - & - \\
\hline WBC & - & - & -0.086 & 0.050 & - & - \\
\hline Hemoglobin & 0.024 & 0.029 & 0.027 & 0.000 & 0.022 & 0.000 \\
\hline Statin agent & -0.832 & 0.029 & -0.269 & 0.271 & -0.119 & 0.537 \\
\hline Hypoglycemic agent & -0.791 & 0.101 & -0.935 & 0.002 & -0.674 & 0.006 \\
\hline Anti-hypertension agent & 0.982 & 0.003 & 0.515 & 0.015 & 0.232 & 0.167 \\
\hline
\end{tabular}

Statistical differences: $P<0.05$; only baseline variables and other interested variables that showed a univariate relationship with aortic diameter with $P<0.1$ were displayed in the table

$B S A$ body surface area, $S B P$ systolic blood pressure, $D B P$ diastolic blood pressure, $C A D$ coronary artery disease, $D M$ diabetes mellitus, $C O P D$ chronic obstructive pulmonary disease, CIMT carotid intima-media thickness, $T C$ total cholesterol, $H D L-C$ high-density lipoprotein cholesterol, $L D L-C$ low-density lipoprotein cholesterol, CRP C-reaction protein, WBC white blood cell

- Not applicable

Table 6 Relationship between the aortic diameters of annulus, sinuses of Valsalva, sinotubular junction and risk factors by multivariate regression analysis

\begin{tabular}{|c|c|c|c|c|c|c|}
\hline \multirow[t]{2}{*}{ Variables } & \multicolumn{2}{|l|}{ Annulus } & \multicolumn{2}{|c|}{ Sinuses of Valsalva } & \multicolumn{2}{|l|}{ Sinotubular } \\
\hline & Coefficient $\beta$ & $P$ values & Coefficient $\beta$ & $P$ values & Coefficient $\beta$ & $P$ values \\
\hline Sex & 0.891 & 0.000 & 2.128 & 0.000 & 1.320 & 0.001 \\
\hline Age, year & -0.019 & 0.014 & - & - & - & - \\
\hline BSA & 1.673 & 0.008 & 3.793 & 0.005 & 2.253 & 0.041 \\
\hline DBP & _- & _- & _- & - & 0.026 & 0.032 \\
\hline Smoking & 0.459 & 0.013 & - & - & - & - \\
\hline Hemoglobin & - & - & 0.029 & 0.044 & - & - \\
\hline
\end{tabular}

Statistical differences: $P<0.05$; only significant variables and data were displayed in the table

$B S A$ body surface area, $D B P$ diastolic blood pressure

- Not applicable 
Table 7 Relationship between the aortic diameters of aortic arch and risk factors by multivariate regression analysis

\begin{tabular}{|c|c|c|c|c|c|c|}
\hline \multirow[t]{2}{*}{ Variables } & \multicolumn{2}{|l|}{ Proximal arch } & \multicolumn{2}{|l|}{ Mid arch } & \multicolumn{2}{|l|}{ Distal arch } \\
\hline & Coefficient $\beta$ & $P$ values & Coefficient $\beta$ & $P$ values & Coefficient $\beta$ & $P$ values \\
\hline Sex & - & - & 0.810 & 0.016 & 0.734 & 0.007 \\
\hline Age, year & - & - & 0.026 & 0.024 & 0.021 & 0.030 \\
\hline $\mathrm{SBP}, \mathrm{mmHg}$ & - & - & -0.019 & 0.002 & - & - \\
\hline $\mathrm{DBP}, \mathrm{mmHg}$ & - & - & 0.046 & 0.000 & 0.038 & 0.000 \\
\hline$C A D$ & -1.064 & 0.000 & -1.068 & 0.000 & -0.478 & 0.017 \\
\hline Hypertension & 0.803 & 0.023 & 0.781 & 0.016 & - & - \\
\hline Hypoglycemic agent & -1.105 & 0.023 & -0.954 & 0.032 & -0.919 & 0.011 \\
\hline
\end{tabular}

Statistical differences: $P<0.05$; only significant variables and data were displayed in the table

$S B P$ systolic blood pressure, $D B P$ diastolic blood pressure, $C A D$ coronary artery disease

- Not applicable

Table 8 Relationship between the aortic diameters of ascending aorta, descending aorta, abdominal aorta and risk factors by multivariate regression analysis

\begin{tabular}{|c|c|c|c|c|c|c|}
\hline \multirow[t]{2}{*}{ Variables } & \multicolumn{2}{|c|}{ Ascending aortaAscending aorta } & \multicolumn{2}{|c|}{ Descending aorta } & \multicolumn{2}{|c|}{ Abdominal aorta } \\
\hline & Coefficient $\beta$ & $P$ values & Coefficient $\beta$ & $P$ values & Coefficient $\beta$ & $P$ values \\
\hline Sex & - & - & _ & _- & 0.845 & 0.001 \\
\hline Age, year & 0.066 & 0.000 & 0.039 & 0.000 & - & - \\
\hline BSA & 3.429 & 0.018 & 3.253 & 0.000 & - & - \\
\hline $\mathrm{DBP}, \mathrm{mmHg}$ & 0.064 & 0.000 & 0.032 & 0.001 & - & - \\
\hline$C A D$ & - & - & - & - & -0.489 & 0.009 \\
\hline Hypertension & _ & _ & 0.661 & 0.030 & _- & _- \\
\hline $\mathrm{HDL}-\mathrm{C}, \mathrm{mmol} / \mathrm{L}$ & 1.348 & 0.042 & _- & _- & 0.947 & 0.004 \\
\hline Hypoglycemic agent & _- & _- & -0.923 & 0.026 & -0.666 & 0.047 \\
\hline
\end{tabular}

Statistical differences: $P<0.05$; only significant variables and data were displayed in the table

$B S A$ body surface area, $D B P$ diastolic blood pressure, $C A D$ coronary artery disease, $H D L-C$ high-density lipoprotein cholesterol, $C R P C$-reaction protein

_ Not applicable

taking antihypertensive medication. In the current study, blood pressure indexes were evaluated at nine sites with small size samples. Our data indicated that DBP was positively associated with the thoracic aorta diameter at several sites but not with abdominal aorta diameter, while SBP was only negatively associated with the diameter of the mid arch. Also, multivariate linear regression analysis excluding aortic dilation confirmed the independent positive association of DBP to ascending aortic expansion. Unlike the limited effects of blood pressure on specific sites (beta $=-0.019$ to 0.064 ), hypertension had considerate effect on aortic diameter of proximal arch, mid arch and descending aorta in our results. Previous studies have observed an independent and significant relationship between hypertension and the increase in the diameter of the abdominal aorta $[15,20]$, but rare study has found the effect of hypertension on the diameter of each segment of the thoracic aorta. Contribution of hypertension and blood pressure in aortic diameter enlargement remains unclear at present. Follow-up studies need to be carried out.

Several studies have found a dose-response relationship between smoking intensity and the risk of AAA [21, 22]. One epidemiological study indicates that smoking history is the strongest factor associated with AAA progression [23]. Mechanistic research report that smoking promotes the degradation of collagen and elastin and consequent weakening of the arterial wall by highly expressed matrix metalloproteinase in aortic wall, finally leading to aortic aneurysm formation [24]. Although we observed that smoking had a robust association with the diameter of almost all segments apart from ascending aorta in the unadjusted model, the strength of this association could not be further demonstrated by fully adjusted analysis. Only the annulus diameter showed its independent association with smoking. The lack of association with abdominal aorta diameter was consistent with previous studies $[7,25]$. Contrarily, some studies 
Table 9 Relationship between risk factors and ascending aortic diameter with no dilation by multivariate linear regression analysis

\begin{tabular}{lcc}
\hline Variables & \multicolumn{2}{c}{ Multivariate linear regression } \\
\cline { 2 - 3 } & Coefficient $\boldsymbol{\beta}$ & $\boldsymbol{P}$ value \\
\hline Sex & -0.083 & 0.847 \\
Age & 0.055 & 0.000 \\
BSA & 2.746 & 0.030 \\
Smoking & -0.346 & 0.355 \\
Alcohol & 0.002 & 0.997 \\
SBP & 0.000 & 0.987 \\
DBP & 0.034 & 0.016 \\
CAD & 0.020 & 0.951 \\
Hypertension & 0.705 & 0.093 \\
DM & -0.174 & 0.721 \\
Hyperlipidaemia & -0.181 & 0.779 \\
Triglyceride, mmol/L & -0.237 & 0.053 \\
HDL-C, mmol/L & 0.526 & 0.355 \\
Lipoprotein (a), mg/L & 0.000 & 0.731 \\
Platelet count & -0.004 & 0.069 \\
Statin agent & -0.392 & 0.220 \\
Anti-hypertension agent & 0.187 & 0.631 \\
Hypoglycemic agent & -0.407 & 0.492 \\
Hemoglobin & 0.013 & 0.256 \\
\hline Sex, age,BSA, smokng, &
\end{tabular}

Sex, age, BSA, smoking, drinking, SBP, DBP, hypertension, DM, CAD, Hyperlipidaemia, triglyceride, HDL-C, LP (a), statin agent, hypoglycemic agent and anti-hypertension agent were considered clinically relevant and were entered into multivariate linear regression analysis of ascending aorta diameter with no dilation, regardless of whether it is significantly related to aortic diameter. Other interested variables that showed a univariate relationship with aortic diameter with $P<0.1$ were included

Statistical differences: $P<0.05$

$B S A$ body surface area, $S B P$ systolic blood pressure, $D B P$ diastolic blood pressure, $C A D$ coronary artery disease, $D M$ diabetes mellitus, $H D L-C$ high-density lipoprotein cholesterol

indicated that incremental widening of the abdominal and ascending aorta was independently related to smoking $[6,20]$. Smoking cessation may have a certain effect on alleviating the dilation of the aorta.

In an unadjusted model, serum lipids were not relevant to aorta diameter of any segment. Surprisingly, HDL-C were an independent determinant of the diameters of ascending and abdominal aorta. Although previous studies on the relation among AAAs, abdominal aortic diameters and serum lipid levels were contradictory [7, 25]. Dyslipidemia has grown in importance among wellestablished risk factors for AAAs. A prospective study cohort for AAA patients found that HDL-C predicted the growth rate of aneurysms for its inverse association with AAA size [26]. Wang et al. [7] found that LDL-C and ratios of TC/HDL-C and LDL-C/HDL-C were independent negative determinants of infrarenal aortic diameter, and infrarenal aortic diameter was significantly positively associated with HDL-C $(\mathrm{r}=0.139, P=0.006)$ independent of age, sex, and height. The current research held the consistent view. Based on the results, we could speculate that HDL-C may have an effect on the increase in the diameter of the ascending aorta and abdominal aorta. The use of statins and the small research samples may affect the accuracy of the outcome. The effect of serum lipids on aortic dilation needs further study.

In contrast, diabetes mellitus plays a protective role in dilated aortic diseases $[8,27]$, characterized by the accumulation of collagen in the aortic wall and subsequent increases in matrix volume [28]. Interestingly, a rat study found that the total count of elastic fibers, fragmentation of the elastic lamina, pericellular matrix deposition, and cell loss/substitution in the tunica media were higher in the diabetic + smoker group (DSG) aorta than those in the smoker group (SG) aorta [24].The negative relationship between the presence of DM and aortic diameter was supported by a few other reports $[8,15]$. However, we found only that DM was inversely related with the diameters of annulus, mid and distal arch in univariate analysis. Consistently, there was an independent negative correlation between the use of hypoglycemic drugs and the aorta diameter of some segments. The effect of blood sugar control on the diameter of the aorta needs further investigation.

Atherosclerosis represents an important independent risk factor for AAA formation [9]. In the Tromsø Study [20], as the measures of subclinical atherosclerosis, coronary artery calcium burden but not CIMT were independently associated with larger aortic diameter, which was supported by a population-based follow-up study [11]. In the current unadjusted analysis, CIMT was only positively associated with descending and abdominal aorta diameter, but was not an independent indicator of any segment. Carotid atherosclerosis, carotid artery stenosis and aortic sclerosis represented no association to all segments' diameters. Differently, coronary artery disease represented a significant independent relationship with the diameter of transverse arch and abdominal aorta in the current multivariate analysis. However, whether this association between atherosclerosis and aortic aneurysm is causal or a result of common shared risk profiles remains unknown. Johnsen et al. [29] indicated that no dose-response relationship between abdominal aortic diameter and atherosclerosis burden assessed as carotid total plaque area, common femoral lumen diameter, and self-reported coronary heart disease, suggested that atherosclerosis may not be a causal event in AAA, but occurred concurrently with aneurysm expansion or secondary to aneurysm expansion.

The segmental inconsistency may be ascribed to distinct structural, genetic, and biochemical factors $[1,30]$. 
Specific segments of the thoracic and abdominal aorta have differences in vascular mechanics, atherosclerotic plaque deposition, MMPs distribution, and cell signaling pathways, which may lead to differences in each segment's susceptibility to risk factors [30].

\section{Limitation}

First, the current study was an observational study in which potential confounders and selection bias could not be fully adjusted. Second, potential misunderstanding due to missing data on use of over-the-counter medication has to be taken into account. Third, the sample size was not large enough and all individuals were included non-randomly resulting in reduced power of the test. Lastly, the current research was biased toward patients with cardiovascular diseases so that some findings may have limited generalizability to non-cardiovascular diseases patients with cardiovascular risk factors. The listed limitations need to be solved by well-designed large observational cohort studies.

\section{Conclusion}

In conclusion, different segments of aortic diameter may have different independent related factors. Aortic segmental diameters were associated with DBP, HDL$\mathrm{C}$, atherosclerosis and other traditional cardiovascular risk factors. These findings may provide new information for understanding the potential mechanism of the early stages of aortic dilation. Additionally, the methods of exploring novel biomarkers for the risk prediction, prevention and early diagnosis of aortic dilation diseases should take segment specificity into consideration. The well-designed studies including cohort studies and molecular level research need further development.

\begin{abstract}
Abbreviations
AAA: Abdominal aortic aneurysm; BSA: Body surface area; TC: Total cholesterol; HDL-C: High-density lipoprotein cholesterol; LDL-C: Low-density lipoprotein cholesterol; CRP: C-reactive protein; WBC: White blood cell; RBC: Red blood cell; CTMT: Carotid intima-media thickness; DM: Diabetes mellitus; SBP: Systolic blood pressure; DBP: Diastolic blood pressure; CAD: Coronary artery disease; COPD: Chronic obstructive pulmonary disease; VIF: Variance inflation factor.
\end{abstract}

\section{Acknowledgements}

Not applicable.

\section{Authors' contributions}

CTT participated in data analysis and manuscript writing. YXA used ultrasound technology to measure aortic diameter, and MP assisted in guiding the measurement of aortic diameter and recording the measured data. FXX and ZY contributed to clinical data collection in the study. WYZ guided the analysis methods of the manuscript. ZHL and WJT contributed to the data analysis and data interpretation. $C X F, J J$ J and TLJ contributed to the design and revised the manuscript. XBH contributed to improving the English writing of the manuscript. All authors read and approved the final manuscript.

\section{Funding}

This study was supported by the grant from National Natural Science Foundation, China (Grant No. 81770475). The funding body assisted in the collection of clinical datas and publication fees.

\section{Availability of data and materials}

The datasets used and/or analyzed during the current study are available from the corresponding author on reasonable request.

\section{Declarations}

Ethics approval and consent to participate

This study was approved by the Ethics Committee of Taizhou Hospital of Zhejiang Province. All participants enrolled in this study have signed informed consent.

\section{Consent for publication}

Not applicable.

\section{Competing interests}

The authors declare no competing interests.

\section{Author details}

${ }^{1}$ Department of Cardiology, Zhejiang Hospital, Hangzhou 310013, Zhejiang Province, China. ${ }^{2}$ Department of Cardiology, Taizhou Hospital Affiliated to Wenzhou Medical University, Linhai 317000, Zhejiang Province, China. ${ }^{3}$ Laboratory of Cardiovascular Disease, Taizhou Hospital Affiliated to Wenzhou Medical University, Linhai 317000, Zhejiang Province, China. ${ }^{4}$ Department of Ultrasonic, Taizhou Hospital Affiliated to Wenzhou Medical University, Linhai 317000, Zhejiang Province, China. ${ }^{5}$ Department of Cardiology, Taizhou Hospital, Zhejiang University School of Medicine, Hangzhou 310013, Zhejiang Province, China. ${ }^{6}$ Department of Surgery, Stanford University School of Medicine, Stanford, CA 94305, USA. ${ }^{7}$ Department of Radiation Oncology, Indiana University School of Medicine, Indianapolis, IN 46202, USA.

Received: 17 August 2020 Accepted: 27 January 2022

Published online: 05 February 2022

\section{References}

1. Kuivaniemi H, Ryer EJ, Elmore JR, Tromp G. Understanding the pathogenesis of abdominal aortic aneurysms. Expert Rev Cardiovasc Ther. 2015;13(9):975-87.

2. Solberg S, Forsdahl SH, Singh K, Jacobsen BK. Diameter of the infrarenal aorta as a risk factor for abdominal aortic aneurysm: the Tromso Study, 1994-2001. Eur J Vasc Endovasc Surg Off J Eur Soc Vasc Surg. 2010;39(3):280-4

3. Freiberg MS, Arnold AM, Newman AB, Edwards MS, Kraemer KL, Kuller $\mathrm{LH}$. Abdominal aortic aneurysms, increasing infrarenal aortic diameter, and risk of total mortality and incident cardiovascular disease events: 10-year follow-up data from the Cardiovascular Health Study. Circulation. 2008;117(8):1010-7.

4. Norman P, Le M, Pearce C, Jamrozik K. Infrarenal aortic diameter predicts all-cause mortality. Arterioscler Thromb Vasc Biol. 2004;24(7):1278-82.

5. Stackelberg O, Wolk A, Eliasson K, Hellberg A, Bersztel A, Larsson SC, et al. Lifestyle and risk of screening-detected abdominal aortic aneurysm in men. J Am Heart Assoc. 2017;6(5):e004725.

6. Cetin M, Kocaman SA, Durakoglugil ME, Erdogan T, Ugurlu Y, Dogan $\mathrm{S}$, et al. Independent determinants of ascending aortic dilatation in hypertensive patients: smoking, endothelial dysfunction, and increased epicardial adipose tissue. Blood Press Monit. 2012;17(6):223-30.

7. Wang JA, Chen XF, Yu WF, Chen H, Lin XF, Xiang MJ, et al. Relationship of heavy drinking, lipoprotein (a) and lipid profile to infrarenal aortic diameter. Vasc Med (Lond, Engl). 2009;14(4):323-9.

8. Tanaka A, Ishii H, Oshima H, Narita Y, Kodama A, Suzuki S, et al. Inverse association between diabetes and aortic dilatation in patients with advanced coronary artery disease. Atherosclerosis. 2015;242(1):123-7. 
9. Elkalioubie A, Haulon S, Duhamel A, Rosa M, Rauch A, Staels B, et al. Metaanalysis of abdominal aortic aneurysm in patients with coronary artery disease. Am J Cardiol. 2015;116(9):1451-6.

10. Glauser F, Mazzolai L, Darioli R, Depairon M. Interaction between widening of diameter of abdominal aorta and cardiovascular risk factors and atherosclerosis burden. Intern Emerg Med. 2014;9(4):411-7.

11. Johnsen SH, Forsdahl SH, Solberg S, Singh K, Jacobsen BK. Carotid atherosclerosis and relation to growth of infrarenal aortic diameter and follow-up diameter: the Tromso Study. Eur J Vasc Endovasc Surg Off J Eur Soc Vasc Surg. 2013;45(2):135-40.

12. Mensel B, Hesselbarth L, Wenzel M, Kuhn JP, Dorr M, Volzke H, et al. Thoracic and abdominal aortic diameters in a general population: MRI-based reference values and association with age and cardiovascular risk factors. Eur Radiol. 2016;26(4):969-78.

13. Tarnoki AD, Tarnoki DL, Littvay L, Garami Z, Karlinger K, Berczi V. Genetic and environmental effects on the abdominal aortic diameter development. Arq Bras Cardiol. 2016;106(1):13-7.

14. Hartshorne TC, McCollum CN, Earnshaw JJ, Morris J, Nasim A. Ultrasound measurement of aortic diameter in a national screening programme. Eur J Vasc Endovasc Surg Off J Eur Soc Vasc Surg. 2011;42(2):195-9.

15. Allison MA, Kwan K, DiTomasso D, Wright CM, Criqui MH. The epidemiology of abdominal aortic diameter. J Vasc Surg. 2008;48(1):121-7.

16. Turkbey EB, Jain A, Johnson C, Redheuil A, Arai AE, Gomes AS, et al. Determinants and normal values of ascending aortic diameter by age, gender, and race/ethnicity in the Multi-Ethnic Study of Atherosclerosis (MESA). J Magn Reson Imaging JMRI. 2014;39(2):360-8.

17. Mao SS, Ahmadi N, Shah B, Beckmann D, Chen A, Ngo L, et al. Normal thoracic aorta diameter on cardiac computed tomography in healthy asymptomatic adults: impact of age and gender. Acad Radiol. 2008;15(7):827-34.

18. Vasan RS, Larson MG, Levy D. Determinants of echocardiographic aortic root size. The Framingham Heart Study. Circulation. 1995;91(3):734-40.

19. Chironi G, Orobinskaia L, Megnien JL, Sirieix ME, Clement-Guinaudeau S, Bensalah $\mathrm{M}$, et al. Early thoracic aorta enlargement in asymptomatic individuals at risk for cardiovascular disease: determinant factors and clinical implication. J Hypertens. 2010;28(10):2134-8.

20. Laughlin GA, Allison MA, Jensky NE, Aboyans V, Wong ND, Detrano R, et al. Abdominal aortic diameter and vascular atherosclerosis: the MultiEthnic Study of Atherosclerosis. Eur J Vasc Endovasc Surg Off J Eur Soc Vasc Surg. 2011;41(4):481-7.

21. Forsdahl SH, Singh K, Solberg S, Jacobsen BK. Risk factors for abdominal aortic aneurysms: a 7-year prospective study: the Tromsø Study, 1994-2001. Circulation. 2009;119(16):2202-8.

22. Pirie K, Peto R, Reeves GK, Green J, Beral V. The 21st century hazards of smoking and benefits of stopping: a prospective study of one million women in the UK. Lancet (Lond, Engl). 2013;381(9861):133-41.

23. Blanchard JF, Armenian HK, Friesen PP. Risk factors for abdominal aortic aneurysm: results of a case-control study. Am J Epidemiol. 2000;151(6):575-83.

24. Barão FTF, Barão VHP, Gornati VC, Silvestre GCR, Silva AQ, Lacchini S, et al. Study of the biomechanical and histological properties of the abdominal aorta of diabetic rats exposed to cigarette smoke. J Vasc Res. 2019:56(5):255-66.

25. Patel AS, Mackey RH, Wildman RP, Thompson T, Matthews K, Kuller L, et al. Cardiovascular risk factors associated with enlarged diameter of the abdominal aortic and iliac arteries in healthy women. Atherosclerosis. 2005;178(2):311-7.

26. Burillo E, Lindholt JS, Molina-Sánchez P, Jorge I, Martinez-Pinna R, BlancoColio LM, et al. ApoA-1/HDL-C levels are inversely associated with abdominal aortic aneurysm progression. Thromb Haemost. 2015;113(6):1335-46.

27. Taimour S, Zarrouk M, Holst J, Rosengren AH, Groop L, Nilsson PM, et al. Aortic diameter at age 65 in men with newly diagnosed type 2 diabetes. Scand CardiovasC J SCJ. 2017;51(4):202-6.

28. Sun H, Zhong M, Miao Y, Ma X, Gong HP, Tan HW, et al. Impaired elastic properties of the aorta in fat-fed, streptozotocin-treated rats. Vasc Remodel Diabet Arteries Cardiol. 2009:114(2):107-13.

29. Johnsen SH, Forsdahl SH, Singh K, Jacobsen BK. Atherosclerosis in abdominal aortic aneurysms: a causal event or a process running in parallel? The Tromsø study. Arterioscler Thromb Vasc Biol. 2010;30(6):1263-8.
30. Ruddy JM, Jones JA, Spinale FG, Ikonomidis JS. Regional heterogeneity within the aorta: relevance to aneurysm disease. J Thorac Cardiovasc Surg. 2008;136(5):1123-30.

\section{Publisher's Note}

Springer Nature remains neutral with regard to jurisdictional claims in published maps and institutional affiliations.
Ready to submit your research? Choose BMC and benefit from:

- fast, convenient online submission

- thorough peer review by experienced researchers in your field

- rapid publication on acceptance

- support for research data, including large and complex data types

- gold Open Access which fosters wider collaboration and increased citations

- maximum visibility for your research: over $100 \mathrm{M}$ website views per year

At BMC, research is always in progress.

Learn more biomedcentral.com/submissions 